\title{
BMJ Open Scandinavian Olecranon Research in the Elderly (SCORE): protocol for a non- inferiority, randomised, controlled, multicentre trial comparing operative and conservative treatment of olecranon fractures in the elderly
}

Ida Rantalaiho (D) , ${ }^{1}$ Inari Laaksonen, ${ }^{1}$ Antti P Launonen, ${ }^{2}$ Toni Luokkala, ${ }^{3}$ Tapio Flinkkilä, ${ }^{4}$ Mikko Salmela, ${ }^{5}$ L Adolfsson, ${ }^{6}$ Bo Olsen, ${ }^{7}$ Kari Isotalo, ${ }^{1}$ Anssi Ryösä, ${ }^{1}$ Ville Äärimaa, ${ }^{1}$ on behalf of the SCORE study group

To cite: Rantalaiho I, Laaksonen I, Launonen AP, et al. Scandinavian Olecranon Research in the Elderly (SCORE): protocol for a non-inferiority, randomised, controlled, multicentre trial comparing operative and conservative treatment of olecranon fractures in the elderly. BMJ Open 2022;12:e055097. doi:10.1136/ bmjopen-2021-055097

- Prepublication history for this paper is available online. To view these files, please visit the journal online (http://dx.doi. org/10.1136/bmjopen-2021055097).

Received 07 July 2021 Accepted 05 January 2022

Check for updates

(c) Author(s) (or their employer(s)) 2022. Re-use permitted under CC BY-NC. No commercial re-use. See rights and permissions. Published by BMJ.

For numbered affiliations see end of article.

Correspondence to

Dr Ida Rantalaiho;

ida.rantalaiho@tyks.fi

\section{ABSTRACT}

Introduction The incidence of olecranon fractures is growing in the elderly population. The traditional operative approach is giving way among the elderly to conservative treatment, which seems to provide a comparable functional outcome with a lower complication burden. However, there is still a lack of reliable evidence to support this shift.

The objective of this trial is to investigate whether conservative treatment of displaced olecranon fractures in patients aged 75 or older yields comparable results to those of operative treatment in terms of pain and daily function.

Methods and analysis Scandinavian Olecranon Research in the Elderly (SCORE) is a randomised, controlled, multicentre, non-inferiority trial. Eligible patients will be randomised to either conservative or operative treatment. The sample size will be 68 patients and allocation done at a 1:1 ratio (34 patients per group). The randomisation is stratified according to the participating hospital and patient's sex. Both groups will receive the same postoperative physiotherapy and pain management. The primary outcome is Disabilities of the Arm, Shoulder and Hand at 1-year follow-up. Secondary outcomes are pain and satisfaction measured on visual analogue scales, Patient Reported Elbow Evaluation, range of motion of the elbow and extension strength of the elbow compared with the unaffected arm. Radiographs will be taken at each follow-up. Primary analysis of the results will be conducted on an intention-to-treat basis.

Ethics and dissemination The study protocol for this clinical trial has been approved by the Ethics Committee of the Hospital District of Southwest Finland and will be submitted for approval to the Regional Ethics Committees in Linköping, Sweden and Copenhagen, Denmark. Every recruiting centre will apply local research approvals. The results of this study will be submitted for publication in peer-reviewed journals.

Trial registration number NCT04401462.
Strengths and limitations of this study

Our study will eventually demonstrate whether conservative treatment can be applied as a first choice to olecranon fractures in the elderly population.

- The multicentre setup with three participating countries increases the generalisability and external validity of this trial.

- The results of this trial are limited to cooperative patients aged 75 years or older, which will limit the external validity of the trial, as a significant proportion of patients in this age group is non-cooperative due to dementia or other comorbidities.

Protocol version This is the second protocol version dated on 16 April 2020.

\section{INTRODUCTION}

Background and rationale

Olecranon fractures account for roughly $1 \%$ of all upper extremity fractures. ${ }^{1}$ Current epidemiological data suggest that the incidence of olecranon fractures is increasing in the elderly population after the seventh decade. ${ }^{12}$ Displaced olecranon fractures have traditionally been treated operatively with osteosynthesis. ${ }^{3}$ The most frequent operative methods for fixating a displaced olecranon fracture are tension band wiring (TBW) and plate fixation (PF). According to previous observational studies, both methods achieve adequate union and function but are also associated with a high rate of reoperations due to operative complications and removal of symptomatic fixation materials after fracture union. ${ }^{4-7}$ Reported reoperation rates 
vary, reaching up to $16 \%-50 \%$ for TBW and $15 \%-33 \%$ for $\mathrm{PF}^{48-11}$

Non-operative, or conservative, treatment has been suggested as a treatment option for elderly patients in whom the function of the injured elbow does not necessarily significantly limit their daily activities. Based on data from a small retrospective series, it seems that conservative treatment could provide a similar functional outcome, with a lower complication burden, for this population. ${ }^{12-14}$ A recent study of a US population reported a $0.66 \%$ annual increase in non-operative management of olecranon fractures in patients aged over 75 years. ${ }^{15}$

To our knowledge, there is only one published and one ongoing randomised study comparing operative and conservative treatment in elderly patients. ${ }^{10} 16$ The published trial was terminated prematurely because of an unacceptably high complication rate in the operative group. ${ }^{10}$ As operative treatment of an isolated displaced olecranon, fracture is still common in the elderly, further research is needed on the role of primary conservative treatment in this patient group.

\section{Objectives and study hypothesis}

The aim of this trial is to study the difference between operative treatment, either with TBW or PF, and conservative treatment of traumatic, displaced (Mayo $2^{17}{ }^{18}$ ) olecranon fractures in the elderly population in a noninferiority study setting. Our null hypothesis is that conservative treatment does not yield inferior outcomes to operative treatment.

\section{Trial design}

SCORE is an ongoing, non-inferiority, randomised, controlled, multicentre trial, with two parallel treatment groups (1:1).

\section{METHODS}

\section{Study setting}

The study protocol is designed in accordance with the SPIRIT 2013 Statement (Standard Protocol Items: Recommendations for Interventional Trials).${ }^{19}$ The trial will be conducted as a multicentre study. The following hospitals participated in designing the study protocol: five university hospitals (Helsinki University Central Hospital, Turku University Central Hospital, Tampere University Hospital, Oulu University Hospital and Kuopio University Hospital) and two regional hospitals (Central-Finland Central Hospital in Jyväskylä and Satakunta Central Hospital in Pori) in Finland, and University Hospitals in Linköping, Sweden and Copenhagen, Denmark. All three countries have a country manager responsible for organising participation locally. Patients will be recruited at the trauma centres of the participating hospitals.

\section{Eligibility criteria}

A member of the study group will assess the eligibility of patients with displaced olecranon fractures referred to

\section{Box 1 Inclusion and exclusion criteria}

\section{Inclusion criteria}

- Radiologically (standard anteroposterior (AP) and lateral radiographs) confirmed, displaced ( $\geq 2 \mathrm{~mm}$ dislocation of the joint surface) fracture of the olecranon.

- Age of patient 75 years or over at time of injury.

\section{Exclusion criteria}

- Delay of more than 2 weeks from traumatic event to day of intervention.

- Mayo type 3 fracture.

- Fracture continuation distal to coronoid process.

- Other acute fracture or nerve damage of ipsilateral upper limb.

- Old fracture (<6 months) or pseudoarthrosis or unhealed nerve injury of ipsilateral upper limb.

- Open fracture.

- Pathological fracture.

- History of alcoholism, drug abuse, psychological or other emotional problems likely to jeopardise informed consent.

- Patient's inability to understand written and spoken Finnish or Swedish or Danish.

- Patient's refusal to participate or cognitive incapability to provide consent.

Patient physically unfit for surgery.

the recruiting centres. Diagnosis will be verified using conventional radiographs (standard anteroposterior (AP) and lateral radiographs). Inclusion and exclusion criteria are listed in box 1 . All eligible patients will be asked to participate in the trial and written informed consent obtained. The two treatment modalities will be openly and carefully explained to the patients at recruitment. All screened patients meeting the inclusion criteria will be recorded.

\section{Interventions}

\section{Operative group}

Patients in the operative group will be prepared for surgery according to the standard of care (plexus and/ or general anaesthesia based on anaesthesiologist's evaluation, antibiotic prophylaxis), and surgery will take place within 2 weeks of the injury. Patients will undergo surgical fixation by the preferred technique of the treating, attending or fellow surgeon (TBW or PF according to Arbeitsgemeinschaft für Osteosynthesefragen -instructions $^{20}$ ) in a manner consistent with the usual protocol of the participating institution. Postoperative protocol will include immobilisation either with a sling or a long-arm plaster splint for 2 weeks followed, by progressive range of motion as tolerated.

\section{Conservative group}

Conservative treatment will consist of a sling and immediate progressive range of motion as tolerated. A long-arm plaster splint may be applied for 2 weeks if needed for pain control and after splint removal active movements will be started as tolerated. 
In both treatment groups, the patients will be referred to physiotherapy at 2 weeks. All patients will be prescribed painkillers, according to local care standards, as needed. Patients will be referred to a ward at their local health centre for rehabilitation if they are unable to manage at home.

\section{Outcomes}

\section{Baseline data}

After enrolment, the following baseline demographics will be recorded: date of birth, sex, date of injury, mechanism of injury, dominant hand, affected side, smoking, possible diabetes or inflammatory arthritis and whether the patient lives in a facility. In addition, a clinical frailty scale $^{21}$ and Disabilities of the Arm, Shoulder and Hand $(\mathrm{DASH})^{22} 23$ questionnaire will be completed at baseline for comparison of the treatment groups. Patients will be asked to answer the DASH questionnaire describing their elbow function within 2 weeks before the injury.

\section{Primary outcome}

Disabilities of the Arm, Shoulder and Hand

The primary outcome compares the DASH ${ }^{22}{ }^{23}$ score at 1 year between treatment groups. DASH is a validated patient-reported outcome measure assessing upper extremity-related deficits and symptoms in daily life. The instrument consists of 30 items, of which at least 27 must be answered for a score to be calculated. The additional four optional items related to work, sports and music (four items each) are discarded in our study. The score ranges from 0 (no disability) to 100 (extreme disability). DASH is available and validated in several languages including Finnish,${ }^{24}$ Swedish ${ }^{25}$ and Danish. ${ }^{26}$ The minimal clinically important difference (MCID) for this questionnaire is 10 points. ${ }^{23} 27$

\section{Secondary outcomes}

Secondary outcomes are both subjective and objective measurements. A full list of secondary outcomes is shown in box 2. Radiographs of the affected arm will also be

\section{Box 2 Outcome measures}

Measurements recorded at 3 and 12 months
Primary outcome measure
DASH at 12 months.
Secondary outcome measures
DASH (other than 12 months).
PREE.
Pain (VAS 0-100).
Satisfaction (VAS 0-100).
ROM of elbow.
Extension strength of elbow compared with unaffected arm (only at
12 months).
Adverse events at any time point.
DASH, Disabilities of the Arm, Shoulder and Hand; PREE, Patient-rated elbow
evaluation; ROM, range of motion; VAS, visual analogue scale.

\section{Box 3 Radiograph evaluation list}

Primary evaluation
Postoperative evaluation
Quality of reduction graded as follows
$\quad-$ Excellent/exact.
- Good/satisfactory (dislocation of joint surface $<2 \mathrm{~mm}$ ).
- Poor (dislocation of joint surface $\geq 2 \mathrm{~mm}$ ).
- Reduction not obtained.
Evaluation of placement of fixation materials.
Evaluation at 2 weeks, 3 and 12 months
Loss of reduction, redisplacement of joint surface $\geq 2 \mathrm{~mm}$ (yes/no).
Failure of fixation (eg, tension band wire broken or out of bone).
In non-operative treatment group, progression of dislocation com-
pared with primary situation.
Signs of bone healing.

taken at each control visit and analysed according to the detailed evaluation list shown in box 3 .

Visual analogue scale; pain and satisfaction

Pain will be assessed on a $0 \mathrm{~mm}$ to $100 \mathrm{~mm}$ visual analogue scale (VAS), from 0 on the left 'no pain' to 100 on the right 'worst possible pain'. VAS is the most frequently used assessment instrument for pain in clinical settings and is structurally simple to use. ${ }^{28}$ Satisfaction with treatment and elbow function will be assessed similarly on a VAS, from 0 on the left 'best possible situation' to 100 on the right 'worst possible situation'.

\section{Patient-rated elbow evaluation}

Patient-rated elbow evaluation (PREE) is an elbow jointspecific measure of pain and disability and is validated with psychometric methods. ${ }^{29}$ The instrument consists of two subsections: pain with 5 items and function with 15. The subsections are computed to weigh pain and disability equally and both are scaled from 0 'best score' to 50 'worst score'. Total score is the sum of subscales. A higher score indicates more pain and functional disability.

\section{Participant timeline}

All patients will have a follow-up appointment at 2 weeks and 3 and 12 months. The detailed schedule for assessments is outlined in table 1 , and the flowchart of the trial is shown in figure 1.

\section{Sample size}

The power calculations are based on assumed behaviour of the DASH questionnaire. The non-inferiority margin was determined to be MCID for this questionnaire, which is 10 points. ${ }^{23}{ }^{27}$ The SD of DASH is assumed to be $15 .{ }^{30}$ Estimated sufficient sample size is based on simple twosample $t$ test with one-sided alternative hypothesis. Using alpha 0.05 and a statistical power of $80 \%$, the power calculations yield a sample size of at least 34 patients per group, taking into an account assumed drop out rate of $20 \%$. 
Table 1 Assessment schedule

\begin{tabular}{|c|c|c|c|c|c|c|c|c|}
\hline \multirow[b]{2}{*}{ Assessment } & \multirow[b]{2}{*}{ ER } & \multirow{2}{*}{$\begin{array}{l}\text { Screening (at local } \\
\text { trauma centre) }\end{array}$} & \multicolumn{2}{|c|}{ Intervention (within 2 weeks of trauma) } & \multicolumn{2}{|l|}{2 weeks } & \multirow{2}{*}{$\begin{array}{l}3 \\
\text { months }\end{array}$} & \multirow{2}{*}{$\begin{array}{l}12 \\
\text { months }\end{array}$} \\
\hline & & & Non-operative & Operative & Non-operative & Operative & & \\
\hline Screening & & $x$ & & & & & & \\
\hline Informed consent & & $x$ & & & & & & \\
\hline $\mathrm{X}$-rays & $\mathrm{x}$ & & & $X^{*}$ & $x$ & $x$ & $\mathrm{X}$ & $\mathrm{X}$ \\
\hline Baseline data & & $x$ & & & & & & \\
\hline Treatment & & & $x$ & $\mathrm{x}$ & & & & \\
\hline Splint removal & & & & & $(X) \dagger$ & $(X) \dagger$ & & \\
\hline Wound review & & & & & & $x$ & & \\
\hline DASH & & & & & & & $\mathrm{X}$ & $\mathrm{X}$ \\
\hline PREE & & & & & & & $x$ & $x$ \\
\hline VAS pain & & & & & & & $x$ & $x$ \\
\hline VAS satisfaction & & & & & & & $\mathrm{x}$ & $\mathrm{x}$ \\
\hline $\begin{array}{l}\text { Adverse event } \\
\text { form } †\end{array}$ & & & & $(X)$ & $(X)$ & $(X)$ & $(X)$ & $(X)$ \\
\hline $\begin{array}{l}\text { Discontinuation } \\
\text { form† }\end{array}$ & & & & & $(X)$ & $(X)$ & $(X)$ & $(X)$ \\
\hline
\end{tabular}

*Postoperatively.

†If required.

DASH, Disabilities of the Arm, Shoulder and Hand questionnaire; PREE, patient-rated elbow evaluation; ROM, range of motion; VAS, visual analogue scale.

\section{Assignment of intervention \\ Allocation}

Randomisation will be stratified according to the participating hospital and sex. The hospitals are grouped for stratification as A: Helsinki, Turku, Pori; B: Tampere, Jyväskylä, Kuopio, Oulu and C: Linköping, Copenhagen. Randomisation will be performed through a web-based online system (https://www.randomize.net/), which gathers the patient information and immediately provides the treatment arm (operative/non-operative). The block size for randomisation is four. Recruitment and randomisation will continue until at least 34 patients are enrolled in each treatment group.

\section{Blinding}

The treatment modalities will be clearly and openly explained to the patients at recruitment. Participants and study investigators will not be blinded to the treatment groups. The statistician will be blinded to the treatment groups and the analysis phase will involve blinded data interpretation.

\section{Declined cohort}

Patients who are otherwise eligible but do not wish to participate, or choose to drop out from the trial, will be asked for permission to conduct a later patient-file follow-up and will be invited to participate in a follow-up study. Informed consent will be obtained from these patients. They will receive the usual care with the treatment method decided by the patient once both treatment methods have been explained. Baseline demographics, treatment modality and the DASH at 1 year will be collected. Analysis of the declined cohort group will be done separately from the randomised controlled trial (RCT) and the results will be compared with those of the RCT.

\section{Patient and public involvement}

Patients were not involved in the design of this study. They will be informed of the results after completion of the study.

\section{DATA MANAGEMENT AND ANALYSIS Data management}

All the data for this study will be collected on trial-specific forms. Patient information forms will be uploaded to a secured cloud server (Sharefile) and the information stored in an electronic research database (RedCap) held at Turku University Hospital, TULES Division, by the study nurse. The study nurse will monitor the data for incomplete items. In case of non-adherence, the 


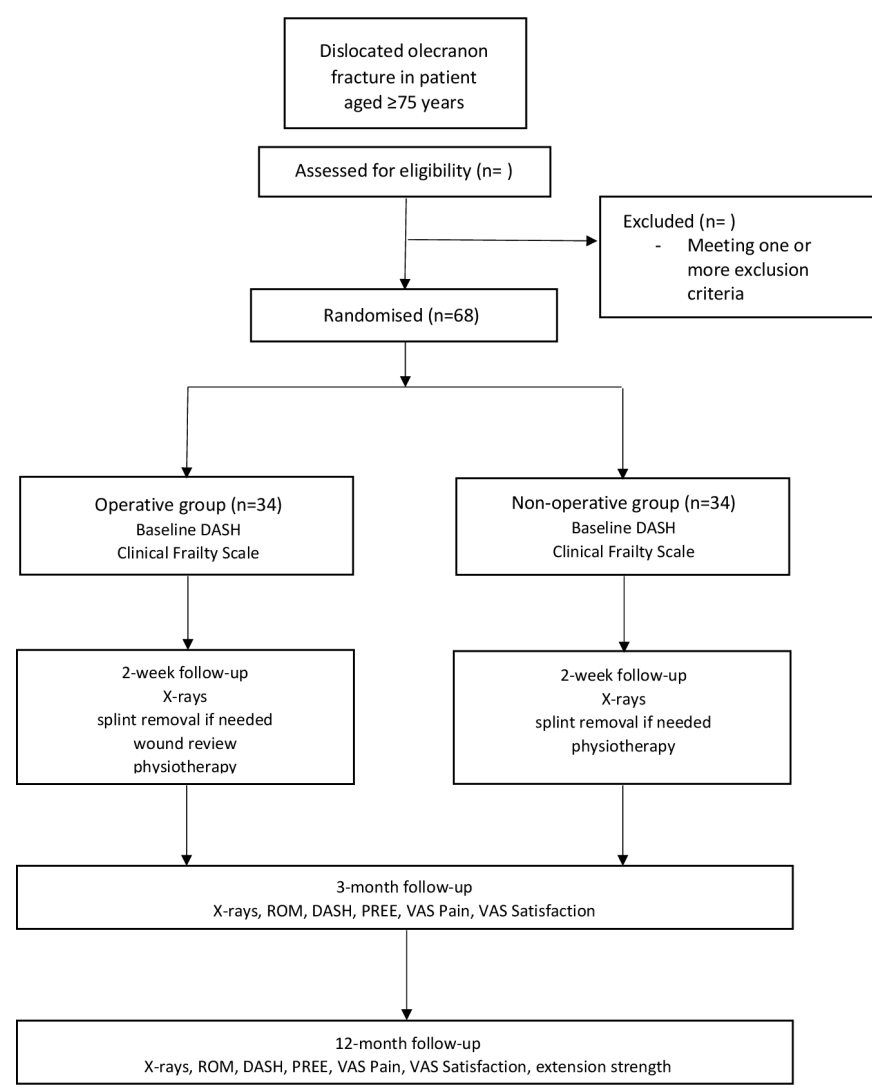

Figure 1 Flowchart of the trial. DASH, Disabilities of the Arm, Shoulder and Hand; PREE Patient-ted Elbow Evaluation; ROM range of motion; VAS visual analogue scale.

investigating physician will be contacted and the reason for non-adherence will be clarified. The RedCap database is protected by access codes known only to the study nurse and one of the investigators. The trial patient data will be stored for 10 years after final follow-up. All the original paper forms are stored securely by a local investigating physician or study nurse. All imaging data are stored in local electronic systems and sent to the study nurse on a compact disc or in electronic format after 1-year follow-up.

\section{Missing items}

Missing data from questionnaires would skew the analyses and, thus, imputation methods will be applied. Missing individual items in DASH and PREE-F are considered missing at random and will be substituted by the average value of other items. If the number of missing values is greater than three, the scores will not be computed. If scores at follow-up are missing or not computable, hot deck imputation will be used where missing score values are substituted by an average score of other patients with similar demographic and baseline data such as age, centre, gender and baseline DASH or PREE-F.

\section{Statistical methods}

After completion of the 2 weeks, 3 months and 1-year follow-up, the data will be analysed by an independent statistician (blinded to the treatment groups). Intention to treat (ITT) will be applied in the analyses. In case of protocol violations, analyses will be carried out for both ITT and per protocol patient populations.

All demographic, preintervention and interventionrelated variables will be tabulated and summarised. All outcome measures will be summarised by visit, and in addition to absolute values, changes relative to baseline values will also be summarised where feasible. Reasons for discontinuation and study duration will be tabulated for all patients by treatment group.

The possibility of multicollinearity between study variables will be investigated in terms of the variance inflation factor. Analysis of the primary outcome measure will be done using generalised linear mixed models (GLMM) suitable for repeated measures with adjusting demographic and intervention-related variables. Autoregressive covariance structure for spatiality of measurement time points is assumed to be suitable in this study setup. GLMM will also be used to analyse secondary outcomes where feasible; otherwise, an alternative analysis method will be selected according to the measurement scale and variable type (eg, independent or paired data and binary, ordinal, nominal or continuous nature). Possible analysis methods that could be used are McNemar's test, the Wilcoxon signed rank test, CochranMantel-Haenszel test, Cochran-Armitage trend test and Jonckheere-Terpstra test.

All results will be presented with 95\% CIs. A one-sided significance level of 0.05 will be used across the analyses. All analyses, tabulation, listings and figures will be done with R V.3.5.2 (R Foundation for Statistical Computing, Vienna, Austria).

\section{Blinded data interpretation}

To diminish interpretation bias, the authors and statistician will be blinded to both treatment groups when analysing the results. The approach involves developing two interpretations of the results based on blinded review of the primary outcome data (treatment A vs treatment B). One interpretation assumes that $\mathrm{A}$ is the operative group, the other that $\mathrm{A}$ is the conservative group. After agreeing that there will be no further changes, the investigators will record their decisions and sign the resulting document. The randomisation code will be then unblinded, the correct interpretation chosen and the manuscript will be finalised. ${ }^{31} 32$

\section{Monitoring}

\section{Data monitoring}

Patient data will be monitored weekly by the study nurse. In case of a delay or interruption in the data, the study nurse will inform the local physician, physiotherapist and the principal investigator.

An interim analysis of the available outcome data will be performed by the trial leader when half the patients have been recruited and treated, to confirm the safety and ethical considerations of the study. In case of significantly more serious adverse events, other than fixation material removal, within any of the treatment modalities, 
premature discontinuation of the study will be considered. Loss of reduction or increase in displacement will not be considered a serious adverse event.

\section{Harms}

Adverse events will be documented throughout the follow-up period at scheduled and non-scheduled clinical visits. Patients and physiotherapists are urged to report any adverse events or health-related issues immediately. In case of any adverse event, the local investigating physician will inform the study nurse and the principal investigator in Turku, Finland. All observed or self-reported adverse events regardless of suspected relationship to the study will be recorded. The local investigating physician will assess the likelihood of the adverse event having been caused by the study treatment on a six-grade causality scale (none, unlikely, possible, probable, definite or cannot be classified). The severity of all adverse events will be graded using the Clavien-Dindo classification. ${ }^{33} 34$ Adverse events in class 3 or higher are considered serious. All adverse events will be dealt with in a symptomatically adequate manner and the patients will be hospitalised if needed.

\section{ETHICS AND DISSEMINATION \\ Ethical approval}

The trial will be conducted according to the revised Declaration of Helsinki by the World Medical Association and the International Council for Harmonisation of Technical Requirements for Pharmaceuticals for Human Use -guidelines for good clinical trial practice. The study has been approved by the Ethics Committee of the Hospital District of Southwest Finland (7/1801/2020) and will be submitted for approval to the local Ethics Committees in Sweden and Denmark. The interventions used in this study are considered safe. Patients are not expected to experience either personal harm or benefit from participating in the trial.

\section{Protocol amendments}

No deviation should be made from the protocol without an amendment. Any amendment affecting patient care must be agreed to by the Scandinavian Olecranon Research in the Elderly (SCORE) study chair (including VÄ, IL, IR, AR, KI and one investigator from each participating centre) and approved by the ethics committees before implementation. If an amendment is administrative only and does not affect patient treatment, it will not require approval by ethics committees, but must be submitted to them for their information.

\section{Consent or assent}

Written informed consent will be obtained by the local recruiting physician at each participating centre. Consent for a patient file follow-up will be obtained from eligible patients who do not wish to participate in the trial.

\section{Confidentiality}

All patient data (paper forms and electronic database) will be handled with confidentiality. During analyses, the patient's personal identification number will be blinded.

\section{Access to data}

The study nurse will maintain the register of treatment groups and patients in the trial. Patient data may be accessed by the principal investigator during the trial in case of adverse events or by the trial leader during interim analyses. After the final 12-month follow-up of all patients, the patient data will be analysed by the principal investigator and author IR, and both analyses and patient data will be accessible to all coinvestigators.

\section{Ancillary and post-trial care}

All patients enrolled in the trial may contact the local treating physician about their treated elbow at any stage of the trial. A patient may withdraw consent and discontinue the study at any time if they wish. Patients will be informed of the trial results by letter after completion of the 1-year follow-up analyses.

\section{Dissemination policy}

The results of this study will be submitted for publication in peer-reviewed journals.

\section{DISCUSSION}

In this SCORE protocol, we describe a non-inferiority, randomised, controlled trial comparing the outcome of conservative treatment of displaced olecranon fractures in the elderly with operative treatment with TBW or PF. We do not aim to demonstrate that conservative treatment is better than the commonly used operative treatment, but to find out whether the results are comparable and sufficient from the patient's perspective, using patient-reported outcome measures. Hence, we chose a non-inferiority setting.

To our knowledge, there is only one ongoing RCT with the same design, ${ }^{16}$ and recently one RCT in Scotland had to be prematurely terminated due to unacceptable complication rates in the operative group. ${ }^{10}$ Loss of reduction was the most frequent complication (6 of 11 ), although it was initially accepted in the conservative group by the study setup. There was no difference in any of the outcome measures between the groups. This data support the need for further research on the role of primary conservative treatment for isolated displaced olecranon fractures in the elderly. In our study, premature discontinuation will be considered if there are significantly more serious adverse events, other than hardware removal, within any of the treatment modalities. It is worth noting that loss of reduction or increase in displacement is not considered a reason for discontinuation, contrary to Duckworth's study.

The evidence to date shows that conservative treatment might provide similar function and pain relief in 
the elderly compared with operative treatment ${ }^{12-14}$ and, therefore, lead to a significantly lower operative and complication burden in this fragile population. Still there is a lack of RCTs and high-quality research on this matter, and no robust conclusion can yet be made. In the literature, populations have been referred to as elderly already in their sixth or seventh decade. ${ }^{134}$ Olecranon fractures in this elderly population are shown to have osteoporotic features. ${ }^{34}$ In reality, health status and everyday functioning abilities vary widely among people in these age groups. Therefore, we chose to raise the inclusion age to 75 to avoid randomising patients who are too functionally active into the conservative treatment group, and, thus, to ensure the ethical aspects of non-operative treatment. Regardless of the good results of conservative treatment, ${ }^{8-10} 12$ it may carry a risk of a symptomatic loss of extension strength, loss of extension range or painful pseudo arthrosis if too much workload is applied to the arm after treatment.

We chose to compare conservative treatment with TBW and PF, as these are globally the most popular surgical methods for olecranon fractures. Several factors direct treatment towards a conservative or operative approach, one of the most important being fracture type. In the SCORE trial, we chose the Mayo classification, ${ }^{35}$ which is simple and easy to use in a clinical setting, to diminish potential bias of the fracture type affecting the outcome. In the trial, we will focus on displaced fractures involving the mid-portion of the olecranon, where the anterior parts of the collateral ligament complexes are intact (Mayo type 2). In these type 2 fractures, ligamentous stability between the upper arm and forearm is thought to be intact, maintaining stability of the elbow regardless of the fracture. ${ }^{17}{ }^{18}$ Each Mayo fracture type is further subdivided into A: non-comminuted and B: comminuted, and fractures in both subgroups will be included in the SCORE trial. Non-displaced Mayo type 1 fractures have widely been safely treated conservatively, and unstable fracture-dislocations (Mayo type 3) should still be treated operatively to regain joint congruency. ${ }^{36}$ We recognise the uncommon risk of Mayo 2 fractures actually being Mayo 3, and subluxation or dislocation of the forearm appearing over the course of non-operative treatment. As this is a potential source of selection bias, we have chosen to follow-up all patients with radiographs at 2 weeks to out rule this phenomenon. In case of dislocation of the forearm, the patients will be treated accordingly.

We chose primarily patient-reported outcome measures, since surgeon-reported outcomes or radiological analyses alone do not provide enough insight into how patients manage their daily life and how satisfied they are with the treatment provided. As the patients determine the success of their treatment, we will be able to distinguish which factors lead to satisfaction or dissatisfaction.

The internal validity of the trial is ensured by minimising bias using an online computer-based randomising system, appropriate statistical testing, blinded data interpretation and an adequate sample size based on power calculation. We consider the external validity of the trial to be good, since inclusion and exclusion criteria are not too numerous, and the results will be compared with the declined cohort results. The results of the trial may be generalised to any other population aged 75 years or older with Mayo type 2, closed olecranon fracture and to younger populations when the fracture shows osteoporotic features, that is, poor bone quality and a low-energy trauma mechanism, and the demands for daily functioning are lowered.

The aim of the SCORE trial is to study whether conservative treatment of displaced olecranon fractures in the elderly population yields sufficient results regarding pain and function without the burden of hospitalisation and complications related to operative treatment.

\section{Author affiliations}

${ }^{1}$ Department of Orthopedics and Traumatology, Turku University Hospital and University of Turku, Turku, Finland

${ }^{2}$ Department of Orthopedics and Traumatology, Tampere University Hospital, Tampere, Finland

${ }^{3}$ Department of Orthopedics and Traumatology, Central Finland Central Hospital, Jyvaskyla, Finland

${ }^{4}$ Department of Orthopedics and Traumatology, Oulu University Hospital, Oulu, Finland

${ }^{5}$ Department of Orthopedics and Traumatology, Helsinki University Hospital, Helsinki, Finland

${ }^{6}$ Institution for Clinical and Experimental Medicine, Division of Orthopaedics, Linköping University, Linkoping, Sweden

${ }^{7}$ Department of Orthopedic Surgery, Herlev and Gentofte Hospital and University of Copenhagen, Copenhagen, Denmark

Acknowledgements We would like to thank our study nurse, Sanna Johansson, and physiotherapist Pekka Karppi from Turku University Hospital, Turku, Finland, for their comments on the study planning.

Collaborators The following persons are part of the SCORE study group: Turku University Hospital (Finland): Sanna Johansson, Pekka Karppi, Tommi Kauko and Milja HolstilaTampere University Hospital (Finland): Bakir Sumrein. Kuopio University Hospital: Simo MiettinenCentral Finland Central Hospital (Finland): Juha Paloneva.

Contributors IL, IR, AR and VÄ developed the trial, IL being the principal investigator and VÄ the trial leader. IR drafted the manuscript and all the members have actively contributed to the further writing and revising the manuscript. $\mathrm{KI}$ is responsible for recruitment of the patients in Turku, and additionally $I R, I L$ and $A R$ assess the eligibility and inclusion of the patients in Turku. AL is responsible for the trial in Tampere, TL in Jyväskylä, TF in Oulu, MS in Helsinki, LA in Linköping and B0 in Copenhagen. All authors have read and approved the final manuscript.

Funding This work was supported by the Finnish Research Foundation for Orthopaedics and Traumatology. Grant number is not applicable.

\section{Competing interests None declared.}

Patient and public involvement Patients and/or the public were not involved in the design, or conduct, or reporting, or dissemination plans of this research.

\section{Patient consent for publication Not applicable.}

Provenance and peer review Not commissioned; externally peer reviewed.

Data availability statement This is a protocol article and no data yet exist. All relevant data will be published in peer-reviewed journal together with the results. Additional anonymised data will be available upon reasonable request.

Open access This is an open access article distributed in accordance with the Creative Commons Attribution Non Commercial (CC BY-NC 4.0) license, which permits others to distribute, remix, adapt, build upon this work non-commercially, and license their derivative works on different terms, provided the original work is properly cited, appropriate credit is given, any changes made indicated, and the use is non-commercial. See: http://creativecommons.org/licenses/by-nc/4.0/. 
ORCID iD

Ida Rantalaiho http://orcid.org/0000-0001-6203-1900

\section{REFERENCES}

1 Duckworth AD, Clement ND, Aitken SA, et al. The epidemiology of fractures of the proximal ulna. Injury 2012;43:343-6.

2 Court-Brown CM, Caesar B. Epidemiology of adult fractures: a review. Injury 2006;37:691-7.

3 Powell AJ, Farhan-Alanie OM, Bryceland JK, et al. The treatment of olecranon fractures in adults. Musculoskelet Surg 2017;101:1-9.

4 De Giacomo AF, Tornetta P, Sinicrope BJ, et al. Outcomes after plating of olecranon fractures: a multicenter evaluation. Injury 2016;47:1466-71.

5 Ren Y-M, Qiao H-Y, Wei Z-J, et al. Efficacy and safety of tension band wiring versus plate fixation in olecranon fractures: a systematic review and meta-analysis. J Orthop Surg Res 2016;11:137.

6 Koziarz A, Woolnough T, Oitment C, et al. Surgical management for olecranon fractures in adults: a systematic review and meta-analysis. Orthopedics 2019;42:75-82.

7 Matar HE, Ali AA, Buckley S. Surgical interventions for treating fractures of the olecranon in adults. Cochrane Database Syst Rev 2014;2014.

8 Chen MJ, Campbell ST, Finlay AK. Surgical and Nonoperative management of olecranon fractures in the elderly: a systematic review and meta-analysis. J Orthop Trauma 2020;35.

9 Duckworth AD, Clement ND, White TO, et al. Plate versus TensionBand wire fixation for olecranon fractures. J Bone Joint Surg 2017;99:1261-73.

10 Duckworth AD, Clement ND, McEachan JE, et al. Prospective randomised trial of non-operative versus operative management of olecranon fractures in the elderly. Bone Joint $J$ 2017;99-B:964-72.

11 Liu Q-H, Fu Z-G, Zhou J-L, et al. Randomized prospective study of olecranon fracture fixation: cable pin system versus tension band wiring. J Int Med Res 2012;40:1055-66.

12 Duckworth AD, Bugler KE, Clement ND, et al. Nonoperative management of displaced olecranon fractures in low-demand elderly patients. J Bone Joint Surg Am 2014;96:67-72.

13 Gallucci GL, Piuzzi NS, Slullitel PAl, et al. Non-Surgical functional treatment for displaced olecranon fractures in the elderly. Bone Joint J 2014;96-B:530-4.

14 Marot V, Bayle-Iniguez X, Cavaignac E, et al. Results of nonoperative treatment of olecranon fracture in over 75-year-olds. Orthop Traumatol Surg Res 2018;104:79-82.

15 Motisi M, Kurowicki J, Berglund DD, et al. Trends in management of radial head and olecranon fractures. Open Orthop J 2017;11:239-47.

16 Symes M, Harris IA, Limbers J, et al. SOFIE: surgery for olecranon fractures in the elderly: a randomised controlled trial of operative versus non-operative treatment. BMC Musculoskelet Disord 2015;16:324.

17 Morrey BF. Current concepts in the treatment of fractures of the radial head, the olecranon, and the coronoid. Instr Course Lect 1995;44:316-27.

18 Morrey BF AR. Fractures of the proximal ulna and olecranon: the elbow and its disorders. Philadelphia: WB Saunders, 1993: 405-28.
19 Chan A-W, Tetzlaff JM, Altman DG, et al. Spirit 2013 statement: defining standard protocol items for clinical trials. Ann Intern Med 2013;158:200-7.

20 Baumgaertner M, Trafton P, Ring D. Ao Foundation surgery reference, 2018. Available: https://surgeryreference.aofoundation. org/orthopedic-trauma/adult-trauma/proximal-forearm/ulna-articularolecranon

21 Rockwood K, Song X, MacKnight C, et al. A global clinical measure of fitness and frailty in elderly people. CMAJ 2005;173:489-95.

22 Hudak PL, Amadio PC, Bombardier C. Development of an upper extremity outcome measure: the DASH (disabilities of the arm, shoulder and hand) [corrected]. The Upper Extremity Collaborative Group (UECG). Am J Ind Med 1996;29:602-8.

23 Gummesson C, Atroshi I, Ekdahl C. The disabilities of the arm, shoulder and hand (DASH) outcome questionnaire: longitudinal construct validity and measuring self-rated health change after surgery. BMC Musculoskelet Disord 2003;4:11.

24 Hacklin E, MAdanat R, Strandberg N. DASH-kyselykaavakkeen suomentaminen JA kulttuuriadaprtaatio. Suom Ortoped Traumatol 2009;32:252-4.

25 Atroshi I, Gummesson C, Andersson B, et al. The disabilities of the arm, shoulder and hand (DASH) outcome questionnaire: reliability and validity of the Swedish version evaluated in 176 patients. Acta Orthop Scand 2000;71:613-8.

26 Lundquist CB, Døssing K, Christiansen DH. Responsiveness of a Danish version of the disabilities of the arm, shoulder and hand (DASH) questionnaire. Dan Med J 2014;61:A4813.

27 Davis AM, Beaton DE, Hudak P, et al. Measuring disability of the upper extremity: a rationale supporting the use of a regional outcome measure. J Hand Ther 1999;12:269-74.

28 Hjermstad MJ, Fayers PM, Haugen DF, et al. Studies comparing numerical rating scales, verbal rating scales, and visual analogue scales for assessment of pain intensity in adults: a systematic literature review. J Pain Symptom Manage 2011;41:1073-93.

29 Vincent JI, MacDermid JC, King GJW, et al. Rasch analysis of the patient Rated elbow evaluation questionnaire. Health Qual Life Outcomes 2015;13:84

30 Hunsaker FG, Cioffi DA, Amadio PC, et al. The American Academy of orthopaedic surgeons outcomes instruments: normative values from the general population. J Bone Joint Surg Am 2002;84:208-15.

31 Gøtzsche PC. Blinding during data analysis and writing of manuscripts. Control Clin Trials 1996;17:285-90.

32 Järvinen TLN, Sihvonen R, Bhandari M, et al. Blinded interpretation of study results can feasibly and effectively diminish interpretation bias. J Clin Epidemiol 2014;67:769-72.

33 Dindo D, Demartines N, Clavien P-A. Classification of surgical complications: a new proposal with evaluation in a cohort of 6336 patients and results of a survey. Ann Surg 2004;240:205-13.

34 Clavien PA, Barkun J, de Oliveira ML, et al. The Clavien-Dindo classification of surgical complications: five-year experience. Ann Surg 2009;250:187-96.

35 Cabanela ME, Morrey BF. The elbow and its disorders. Philadelphia, PA, USA: WB Saunders, 1993.

36 Buijze G, Kloen P. Clinical evaluation of locking compression plate fixation for comminuted olecranon fractures. J Bone Joint Surg Am 2009;91:2416-20.

37 Hak DJ, Golladay GJ. Olecranon fractures: treatment options [In Process Citation]. J Am Acad Orthop Surg 2000;8:266-75. 